\title{
A prática da terapia ocupacional junto à população infantil: revisão bibliográfica do período de 1999 a 2009*
}

\section{The practice of occupational therapy with the child population: literature review of the period from 1999 to 2009}

\author{
Mariana Leme Gomes ${ }^{1}$, Fátima Corrêa Oliver²
}

GOMES, M. L.; OLIVER, F. C. A prática da terapia ocupacional junto à população infantil: revisão bibliográfica do período de 1999 a 2009. Rev. Ter. Ocup. Univ. São Paulo, v. 21, n. 2, p. 121-129, maio/ago. 2010.

\begin{abstract}
RESUMO: Trata-se de estudo sobre o trabalho desenvolvido em terapia ocupacional junto a crianças no Brasil. Foi realizado um levantamento bibliográfico de artigos publicados entre 1999 e 2009 nas bases de dados Literatura Latino-Americana e do Caribe de Ciências da Saúde e Scientific Eletronic Library Online e no periódico Cadernos de Terapia Ocupacional da UFSCar. O material foi organizado e analisado em relação a autoria, ano e periódico de publicação, tipo de trabalho, metodologia/modelo de atenção, problemática e instituição/local de atuação. Foram localizados 84 artigos, a maior parte vinculada a autores das universidades da região sudeste. Os trabalhos com crianças são desenvolvidos nos campos da saúde, educação e assistência social. Os relatos de experiência e artigos de pesquisa foram os tipos de trabalhos mais divulgados. As metodologias de estudo empregadas nas pesquisas são relativas a estudos descritivos e observacionais e ensaios clínicos. As intervenções elencadas nos relatos de experiência são atendimentos individuais e em grupo e uso de atividades lúdicas, entre outras. Foram elencadas 12 problemáticas e as mais estudadas foram a atenção à criança em situação de risco social, em processo de hospitalização, além de temas como inclusão escolar, brincar, modelos e instrumentos de avaliação e atividades e recursos terapêuticos. O estudo possibilitou conhecer o panorama nacional da terapia ocupacional com a população infantil a partir do olhar e das estratégias de estudo escolhidas pelos seus autores.
\end{abstract}

DESCRITORES: Terapia ocupacional/tendências; Terapia ocupacional/métodos; Literatura de revisão como assunto; Guia de prática clínica; Criança; Desenvolvimento infantil; Defesa da criança e do adolescente.

* Esse estudo é parte do processo de avaliação das disciplinas MFTO0213 - Iniciação à Pesquisa I e MFTO0254 - Iniciação à pesquisa II Terapia Ocupacional e Saúde da Pessoa com Deficiência, do Curso de Graduação em Terapia Ocupacional do Departamento de Fisioterapia, Fonoaudiologia e Terapia Ocupacional da FMUSP.

1. Discente do Curso de Terapia Ocupacional do Departamento de Fisioterapia, Fonoaudiologia e Terapia Ocupacional da FMUSP, no período de 2006-2010.

2. Docente do Curso de Terapia Ocupacional do Departamento de Fisioterapia, Fonoaudiologia e Terapia Ocupacional da FMUSP.

Endereço para correspondência: Rua Cipotânea, 51. Cidade Universitária. CEP 05360-160 - SP. E-mail: fcoliver@usp.br 


\section{INTRODUÇÃO}

té o século XVII as crianças eram vistas
como pequenos adultos, não recebiam qual-
quer cuidado especial e tinham um índice de mortalidade muito alto (AIRÉS, 1981). Com o avanço do conhecimento sobre a infância, na pediatria, psicologia e pedagogia, foi possível desvelar especificidades do desenvolvimento infantil e dedicar maior atenção à assistência e à proteção das crianças.

A terapia ocupacional é também uma área que desenvolve um importante trabalho com essa população e procura contribuir para seu desenvolvimento, autonomia e participação na vida social.

Mota e Takatori (2001) apontam que a população infantil atendida em terapia ocupacional inclui recém-nascidos, bebês e crianças que apresentam riscos ou alterações no seu desenvolvimento, decorrentes de circunstâncias de ordem orgânica, emocional e/ou social, que podem acontecer antes, durante e logo após o nascimento ou ainda durante a infância.

$\mathrm{Na}$ literatura, os temas da infância estudados por terapeutas ocupacionais são a atenção às crianças hospitalizadas, ao neonato de risco, às crianças com deficiência intelectual, com transtorno emocional, com transtorno de coordenação, com desordens neuromotoras e com atraso no desenvolvimento. Há ainda estudos sobre crianças em situação de risco social, além da inclusão escolar e da importância do brincar (KUDO et al, 1990; CAVALCANTI; GALVÃO, 2007; AOKI et al., 2006).

O terapeuta ocupacional pode utilizar na atenção à criança atividades lúdicas, expressivas e criativas como recursos terapêuticos e também a indicação de equipamentos assistivos, visando desenvolver e/ou ainda descobrir novas habilidades (CARLO; BARTALOTTI, 2001).

Este estudo pretende conhecer, os trabalhos desenvolvidos por terapeutas ocupacionais junto a crianças no Brasil e contribuir para o reconhecimento das tendências atuais de estudos e da atenção a essa população entre os profissionais da área.

\section{Procedimentos metodológicos}

Trata-se de um estudo de revisão bibliográfica de artigos, pois essa produção tem sido crescente e pode representar as preocupações dos profissionais tanto da pesquisa e ensino como daqueles relacionados à assistência. Os artigos também são materiais de divulgação e disseminação de conhecimentos e de práticas assistenciais em curso.

Foi organizado um levantamento bibliográfico de artigos de terapia ocupacional na atuação com crianças no
Brasil, entre 1999 e 2009 nas bases de dados Literatura Latino-Americana e do Caribe de Ciências da Saúde (LILACS) e Scientific Eletronic Library Online (SCIELO), com os descritores "terapia ocupacional" e "criança", "terapia ocupacional" e "infância" e "terapia ocupacional" e "desenvolvimento infantil". Foram analisados artigos de periódicos indexados e não indexados, no caso do Cadernos de Terapia Ocupacional da UFSCar, que não tem mecanismos automáticos de busca, foi feita uma varredura manual. Até o momento do levantamento bibliográfico, a Revista de Terapia Ocupacional da USP não havia disponibilizado os números relativos ao ano de 2009. Alguns artigos estavam disponibilizados na integra na rede internacional de computadores e outros foram localizados em bibliotecas.

Foram levantados 85 artigos e apenas um deles não foi localizado. As informações foram organizadas em um banco de dados e posteriormente analisadas, em relação à autoria, ano e periódico de publicação, tipo de trabalho, metodologia/modelo de atenção, problemática abordada e instituição/local de atuação.

\section{A produção bibliográfica segundo periódicos, instituição,} localidade e ano de publicação

Os artigos levantados foram publicados em 14 periódicos (Tabela 1). O Cadernos de Terapia Ocupacional de UFSCar e a Revista de Terapia Ocupacional da USP, responsáveis por $52 \%$ das publicações, são específicos de terapia ocupacional e organizados por duas grandes Universidades. A partir do ano de 2006, alguns artigos foram publicados em periódicos de Oftalmologia, Pediatria e Oncologia, o que representa uma tendência de ampliação do campo de atuação da terapia ocupacional e da divulgação de seu trabalho em outras áreas do cuidado da infância.

A produção dos artigos está concentrada entre as universidades do País, que são responsáveis por $74 \%$ deles. Entre as demais instituições (15), apenas a Associação de Assistência à Criança Deficiente (AACD) publicou 3 artigos, o que indica que a produção científica está essencialmente vinculada às instituições de ensino.

Mesmo entre as universidades há desigualdade na produção. A Universidade Federal de São Carlos e a Universidade de São Paulo divulgaram 20 e 13 artigos respectivamente, seguidas pela Universidade Federal de Minas Gerais, com 6 artigos e a Universidade de São Paulo - Campos Ribeirão Preto com 5 artigos. Outras 12 instituições de ensino não apresentam números significativos de artigos. Manteve-se assim a tendência de concentração da produção bibliográfica na região sudeste, com 70 artigos, seguida da região nordeste com 5 , a região norte com 4 e a região sul com 3 . 
GOMES, M. L.; OLIVER, F. C. A prática da terapia. Rev. Ter. Ocup. Univ. São Paulo, v. 21, n. 2, p. 121-129, maio/ago. 2010.

Tabela 1 - Artigos publicados, segundo periódico e ano de publicação

\begin{tabular}{|c|c|c|c|c|c|c|c|c|c|c|c|c|}
\hline \multirow[t]{2}{*}{ Nome do Periódico } & \multicolumn{12}{|c|}{ Ano } \\
\hline & 2000 & 2001 & 2002 & 2003 & 2004 & 2005 & 2006 & 2007 & 2008 & 2009 & Total & $\%$ \\
\hline Cadernos de Terapia Ocupacional da UFSCar & 2 & 4 & 4 & 1 & 1 & 7 & 6 & 6 & 5 & 4 & 40 & 47,6 \\
\hline $\begin{array}{l}\text { Revista de Terapia Ocupacional da Universidade de São } \\
\text { Paulo }\end{array}$ & 1 & 1 & - & 2 & 2 & - & 7 & 2 & 2 & - & 17 & 20,2 \\
\hline Temas sobre Desenvolvimento & - & 1 & 4 & 1 & 1 & 2 & 2 & - & 3 & - & 14 & 16,6 \\
\hline O Mundo da Saúde & - & - & 1 & - & - & - & - & - & 1 & - & 2 & 2,4 \\
\hline Revista Brasileira de Saúde Materno Infantil & - & - & - & - & - & - & - & 1 & 1 & - & 2 & 2,4 \\
\hline Arquivos Bras. de Oftalmologia & - & - & - & - & - & - & 1 & - & - & - & 1 & 1,2 \\
\hline Estilo da Clínica & - & - & - & - & - & - & - & 1 & - & - & 1 & 1,2 \\
\hline Estudos de Psicologia (Campinas) & - & - & - & - & - & - & 1 & - & - & - & 1 & 1,2 \\
\hline Pediatria São Paulo & - & - & - & - & - & - & - & 1 & - & - & 1 & 1,2 \\
\hline Psicologia Ciência e Profissão & - & - & - & - & - & - & - & 1 & - & - & 1 & 1,2 \\
\hline Revista Brasileira de Cancerologia & - & - & - & - & - & - & 1 & - & - & - & 1 & 1,2 \\
\hline $\begin{array}{l}\text { Revista Brasileira do Crescimento e Desenvolvimento } \\
\text { Humano }\end{array}$ & - & - & - & - & - & - & - & - & 1 & - & 1 & 1,2 \\
\hline Revista Brasileira de Psiquiatria & - & - & - & - & - & - & - & - & 1 & - & 1 & 1,2 \\
\hline Revista Paulista de Pediatria & - & 1 & - & - & - & - & - & - & - & - & 1 & 1,2 \\
\hline Total & 3 & 7 & 9 & 4 & 4 & 10 & 18 & 12 & 14 & 4 & 84 & 100 \\
\hline
\end{tabular}

Nesse sentido, Galheigo e Russo (2008) discutem essa desigualdade quando comparam a produção bibliográfica em práticas hospitalares com o número de profissionais e de cursos de terapia ocupacional por regiões e concluem que o pequeno número de artigos publicados pode estar mais relacionado ao pouco incentivo à pesquisa e à produção bibliográfica do que à baixa inserção de terapeutas ocupacionais em atividades assistenciais nas demais regiões.

Tanto a prática de intervenção com crianças como a pesquisa são muitas vezes realizadas pelas universidades em parceria com outros serviços do território ou em hospitais e ambulatórios próprios. Grande parte dos trabalhos localizados acontece em hospitais, ambulatórios, centros de reabilitação, escolas, creches, espaços comunitários, abrigos e organizações não governamentais (ONGs).

As práticas não são desenvolvidas unicamente no âmbito das instituições de saúde, mas também nos campos da educação e da assistência social. Nesse sentido, observa-se o que Barros et al. (2002) discutem: o terapeuta ocupacional é posto em questão e percebe que sua atuação se inscreve em um processo histórico e contextual, de dimensões técnica e política inseparáveis e que as problemáticas das pessoas que acompanha não se reduzem ao eixo estruturador saúdedoença, e então busca desenvolver um campo de intervenção e de estudos que extrapola os limites predefinidos pela prática assistencial em saúde.

\section{A produção bibliográfica segundo autoria, problemática e tipo de trabalho}

Os 84 artigos foram produzidos por 179 autores, nem todos terapeutas ocupacionais, uma vez que alguns trabalhos são interdisciplinares. A maioria dos autores $(\mathrm{n}=154)$ escreveu um artigo, outros 15 têm duas produções cada um. Entre os autores que mais têm publicado sobre o tema, 5 deles produziram 3 artigos, 3 produziram 4 artigos e apenas 2 produziram 5 artigos. .9

As problemáticas mais estudadas são a atenção às crianças em situação de risco social, à criança em processo de adoecimento e hospitalização e a inclusão escolar, que apesar de serem observadas anteriormente, passam a ser tendência a partir de 2006, coincidindo com o aumento da produção de artigos (Tabela 2). Alguns temas, como a atenção à família da criança com deficiência, à criança com deficiência visual e a intervenção em educação infantil foram mais discutidos a partir de 2003 e 2005, o que pode refletir tanto uma expansão do campo de atuação da terapia ocupacional, como uma tendência dos profissionais produzirem mais trabalhos científicos sobre a sua prática. 
GOMES, M. L.; OLIVER, F. C. A prática da terapia. Rev. Ter. Ocup. Univ. São Paulo, v. 21, n. 2, p. 121-129, maio/ago. 2010.

Tabela 2 - Artigos publicados, segundo problemática e ano de publicação

\begin{tabular}{|c|c|c|c|c|c|c|c|c|c|c|c|c|}
\hline Problemática & & & & & & Ano & & & & & & \\
\hline & 2000 & 2001 & 2002 & 2003 & 2004 & 2005 & 2006 & 2007 & 2008 & 2009 & Total & $\%$ \\
\hline Atenção a criança em situação de risco social & 2 & 1 & 1 & 1 & - & 2 & 4 & 1 & - & - & 12 & 14,3 \\
\hline $\begin{array}{l}\text { Atenção à criança em processo de adoecimento } \\
\text { e hospitalização }\end{array}$ & - & 2 & - & - & - & 1 & 2 & - & 4 & 1 & 10 & 11,9 \\
\hline Inclusão escolar & - & 1 & 1 & 1 & 1 & - & 1 & 3 & 2 & - & 10 & 11,9 \\
\hline Modelos e Avaliações em TO & - & 1 & 2 & - & 2 & - & 1 & - & 2 & 1 & 9 & 10,7 \\
\hline O brincar & - & 2 & 1 & - & 1 & - & 2 & 2 & - & - & 8 & 9,6 \\
\hline Atenção à criança com deficiência visual & - & - & - & 1 & - & 1 & 2 & 1 & 1 & - & 6 & 7,1 \\
\hline Uso de atividades e recursos terapêuticos & - & - & 2 & - & - & 1 & 1 & 1 & - & 1 & 6 & 7,1 \\
\hline $\begin{array}{l}\text { Atenção à criança com transtorno mental e/ou } \\
\text { deficiência intelectual }\end{array}$ & - & - & - & - & - & - & 3 & 1 & 1 & - & 5 & 5,9 \\
\hline $\begin{array}{l}\text { Atenção à } \\
\text { criança com deficiência física }\end{array}$ & 1 & - & 1 & - & - & - & 1 & - & 1 & - & 4 & 4,8 \\
\hline Intervenção em educação infantil & - & - & - & - & - & 2 & - & 1 & - & - & 3 & 3,6 \\
\hline $\begin{array}{l}\text { Atenção às crianças em situação de abriga- } \\
\text { mento }\end{array}$ & - & - & - & 1 & - & 1 & - & 1 & - & - & 3 & 3,6 \\
\hline $\begin{array}{l}\text { Atenção à } \\
\text { família de crianças com deficiência }\end{array}$ & - & - & - & - & - & 1 & - & - & 2 & - & 3 & 3,6 \\
\hline Outros & - & - & 1 & - & - & - & 1 & 1 & 1 & 1 & 5 & 5,9 \\
\hline Total & 3 & 7 & 9 & 4 & 4 & 9 & 18 & 12 & 14 & 4 & 84 & 100.0 \\
\hline
\end{tabular}

Não foi possível conhecer a faixa etária das crianças atendidas, pois a maior parte dos autores utilizou intervalos etários diferentes e outros não explicitaram essa informação. No entanto, nota-se que há trabalhos realizados com crianças em todas as fases de desenvolvimento.

Relatos de experiência, artigos de pesquisa e discussões teóricas são os tipos de trabalho mais comuns (Gráfico 1).

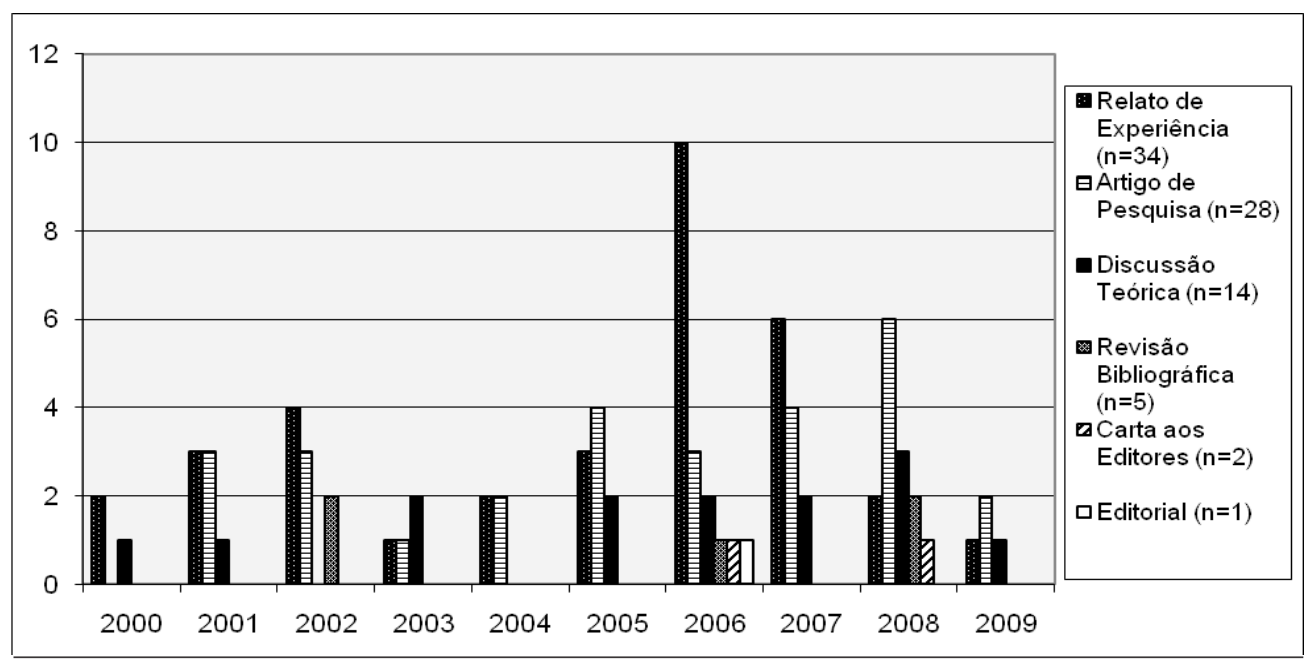

Gráfico 1 - Artigos publicados, segundo tipo de trabalho e ano de publicação 
GOMES, M. L.; OLIVER, F. C. A prática da terapia. Rev. Ter. Ocup. Univ. São Paulo, v. 21, n. 2, p. 121-129, maio/ago. 2010.

Os Quadros 1 e 2 apresentam sinteticamente as problemáticas mais discutidas, os autores principais, os tipos de trabalhos e as metodologias de estudo e intervenções utilizadas.

Quadro 1 - Artigos publicados, segundo problemática, autores, tipo de trabalho ${ }^{1}$ e metodologia empregada ${ }^{2}$, 1999-2009

\begin{tabular}{|c|c|c|c|c|}
\hline \multirow[b]{2}{*}{ Problemáticas } & \multirow[b]{2}{*}{ Autores } & \multicolumn{3}{|c|}{ Metodologia empregada por tipo de trabalho } \\
\hline & & Relato de experiência & Artigo de pesquisa & Revisão Bibliográfica \\
\hline $\begin{array}{l}\text { Atenção à crian- } \\
\text { ças em situação } \\
\text { de risco social }\end{array}$ & $\begin{array}{l}\text { - Ana Paula Malfitano; } \\
\text { - Roseli Esquerdo Lopes; } \\
\text { - Denise Dias Barros; } \\
\text { - Débora Galvani; } \\
\text { - Lucivaldo Araújo; } \\
\text { - Umaia El-Khatib. }\end{array}$ & $\begin{array}{l}\mathrm{n}=7 \\
\text { - Atendimentos em grupos ou individuais } \\
\text { - Uso de atividades lúdicas e expressivas } \\
\text { - Passeios }\end{array}$ & $\begin{array}{l}\mathrm{n}=2 \\
\text { - Pesquisas com dados } \\
\text { secundários } \\
\text { - Estudo descritivo com } \\
\text { aplicação de questionários }\end{array}$ & \\
\hline $\begin{array}{l}\text { Atenção à criança } \\
\text { em processo de } \\
\text { adoecimento e } \\
\text { hospitalização }\end{array}$ & $\begin{array}{l}\text { - Luiza Iara Pfeifer; } \\
\text { - Cláudia Maria Simões } \\
\text { Martinez; } \\
\text { - Sandra Galheigo; } \\
\text { - Andréa Amparo. }\end{array}$ & $\begin{array}{l}\text { n=4 } \\
\text { - Avaliação inicial das crianças, a partir } \\
\text { de entrevistas ou observações } \\
\text { - Atendimentos individuais ou em grupo } \\
\text { - Uso atividades lúdicas } \\
\text { - Trabalho multidisciplinar } \\
\text { - Programas de apoio às famílias }\end{array}$ & $\begin{array}{l}\mathrm{n}=4 \\
\text { - Estudo descritivo com } \\
\text { aplicação de questionários. }\end{array}$ & \\
\hline $\begin{array}{l}\text { Atenção a } \\
\text { crianças com } \\
\text { deficiência visual, } \\
\text { física, intelectual } \\
\text { e com transtorno } \\
\text { mental. }\end{array}$ & $\begin{array}{l}\text { - Raquel Costa Abulquer- } \\
\text { que; } \\
\text { - Andréa Jurdi; } \\
\text { - Maria Inês Brunello; } \\
\text { - Marisa Mancini; } \\
\text { - Marina Palhares }\end{array}$ & $\begin{array}{l}\mathrm{n}=6 \\
\text { - Avaliação inicial (entrevistas, observa- } \\
\text { ção e uso de instrumentos de avaliação) } \\
\text { - Atendimentos individuais ou em grupo } \\
\text { - Uso de atividades lúdicas e expressivas } \\
\text { - Manipulação corporal, massagem e } \\
\text { relaxamento } \\
\text { - Visitas domiciliares } \\
\text { - Passeios } \\
\text { - Intervenção na escola }\end{array}$ & $\begin{array}{l}\mathrm{n}=2 \\
\text { - Pesquisas com dados } \\
\text { secundários } \\
\text { - Estudos descritivos com } \\
\text { aplicação de questionários } \\
\text { - Estudo observacional }\end{array}$ & $\begin{array}{l}\mathrm{n}=1 \\
\text { - Bases de Dados: } \\
\text { Ovid, Medline, CI- } \\
\text { NAHL e Lilacs } \\
\text { - Descritores: terapia } \\
\text { ocupacional, paralisia } \\
\text { cerebral e criança } \\
\text { - Avaliação da metodo- } \\
\text { logia de pesquisa com } \\
\text { a escala OTSeeker }\end{array}$ \\
\hline
\end{tabular}

${ }^{1}$ Classificação baseada em Vieria e Hossne (2001).

${ }^{2}$ Os estudos teóricos estão apresentados ao longo do texto do artigo.

${ }^{3} \mathrm{O}$ agrupamento dessas problemáticas foi realizado por semelhança na metodologia de abordagem dos problemas dos grupos.

Nos trabalhos sobre atenção à criança em situação de risco social, El-Khatib (2005) aponta que a expressão "situação de risco social" não se refere à criança, mas à condição na qual ela se encontra. Malfitano et al. (2006) complementam que as vulnerabilidades são diversas, entre elas, violência doméstica, negligência de cuidados, evasão escolar, falta de aportes sócio-afetivos e uso abusivo de substâncias psicoativas.

De acordo com Barros et al. (2001), não se deve fazer uma leitura superficial e reducionista dessa problemática, que culpabilize as crianças individualmente, mas considerála como dependente de fatores complexos da sociedade contemporânea. Os autores apontam ainda que enfrentar essa problemática requer a construção de um novo conceito de cidadania, uma vez que mesmo com 20 anos de aprovação do Estatuto da Criança e do Adolescente essa população ainda não é reconhecida como sujeitos de direito. Visto isso, as ações de terapia ocupacional devem possibilitar o fortalecimento das redes de sustentabilidade e de suporte relacional, econômico e afetivo e o apoio ao protagonismo, à cidadania e ao exercício de direitos das crianças (BARROS et al., 2001; MALFITANO et al., 2006).

Em relação à atenção à criança em processo de adoecimento e hospitalização Galheigo e Amparo (2008) consideram que a situação de internação hospitalar é causadora de rupturas na vida cotidiana dos sujeitos e que, no caso das crianças, esse impacto pode gerar maiores dificuldades, tendo em vista, sua condição de processo de crescimento, sua dependência do cuidado do outro, o pouco repertório para lidar com a infinidade de situações e exposições a que estão submetidas durante a internação. Para as autoras, a terapia ocupacional trabalha com a intenção de contribuir para a constituição de estratégias de cuidado e de enfrentamento das dificuldades vivenciadas por elas em suas ações e cotidianos. 
GOMES, M. L.; OLIVER, F. C. A prática da terapia. Rev. Ter. Ocup. Univ. São Paulo, v. 21, n. 2, p. 121-129, maio/ago. 2010.

Quadro 2 - Artigos publicados segundo problemática, autores, tipo de trabalho e metodologia empregada ${ }^{2}$, 1999-2009

\begin{tabular}{|c|c|c|c|c|}
\hline \multirow[b]{2}{*}{ Problemática } & \multirow[b]{2}{*}{ Autores } & \multicolumn{3}{|c|}{ Metodologia empregada por tipo de trabalho } \\
\hline & & Relato de experiência & Artigo de pesquisa & Revisão bibliográfica \\
\hline $\begin{array}{l}\text { Inclusão } \\
\text { Escolar }\end{array}$ & $\begin{array}{l}\text { - Andréa Jurdi; } \\
\text { - Eucenir Rocha; }\end{array}$ & $\begin{array}{l}\text { n=6 } \\
\text { - Observação do funcionamento } \\
\text { institucional } \\
\text { - Reuniões para a discussão, orien- } \\
\text { tação e capacitação de funcionários } \\
\text { - Reuniões para a sensibilização da } \\
\text { comunidade } \\
\text { - Grupos de atividades lúdicas com } \\
\text { as crianças }\end{array}$ & $\begin{array}{l}\mathrm{n}=2 \\
\text { - Estudo descritivo com } \\
\text { aplicação de questionários } \\
\text { - Estudo de caso controle } \\
\text { - Pesquisas com dados } \\
\text { secundários }\end{array}$ & \\
\hline $\begin{array}{l}\text { Modelos e } \\
\text { instrumentos } \\
\text { de avaliação } \\
\text { em terapia } \\
\text { ocupacional }\end{array}$ & $\begin{array}{l}\text { - Fabiana Frigeri } \\
\text { Vitta; } \\
\text { - Marisa Mancini; } \\
\text { - Beatriz Galvão }\end{array}$ & & $\begin{array}{l}\mathrm{n}=6 \\
\text { - Estudo observacional } \\
\text { - Ensaios clínicos com } \\
\text { grupo controle ou com } \\
\text { indivíduo como seu próprio } \\
\text { controle }\end{array}$ & $\begin{array}{l}\mathrm{n}=2 \\
\text { - Bases de Dados: Medline e OTDBase } \\
\text { - Descritores: developmental coordina- } \\
\text { tion disorder, sense of self-efficacy, } \\
\text { perceived self-efficacy, child and oc- } \\
\text { cupational therapy } \\
\text { - Pesquisas em periódicos, e sites ( BI- } \\
\text { REME e Associação Americana de TO) }\end{array}$ \\
\hline O Brincar & $\begin{array}{l}\text { - Luiza lara } \\
\text { Pfeifer; } \\
\text { - Marisa Takatori; } \\
\text { - Edda Bomtem- } \\
\text { po; } \\
\text { - Maria Luiza } \\
\text { Emel }\end{array}$ & $\begin{array}{l}\mathrm{n}=3 \\
\text { - Atendimentos individuais ou em } \\
\text { grupo; } \\
\text { - Uso de atividades lúdicas como } \\
\text { recurso terapêutico }\end{array}$ & $\begin{array}{l}\mathrm{n}=2 \\
\text { - Estudo observacional } \\
\text { - Ensaios clínicos com } \\
\text { grupo controle } \\
\text { - Estudo descritivo com } \\
\text { aplicação de questionários } \\
\text { - Registro a partir de ima- } \\
\text { gens de vídeo e fotografia. }\end{array}$ & $\begin{array}{l}\text { n=1 } \\
\text { - Bases de Dados: Scielo e Lilacs, e } \\
\text { periódicos de acesso livre na CAPES } \\
\text { - Descritores: brinquedoteca, brincar, } \\
\text { atividade lúdica, hospitalização, infân- } \\
\text { cia, criança. } \\
\text { - Pesquisas nas bibliotecas da USP, } \\
\text { em anais de congressos e no site da } \\
\text { ferramenta acadêmica Google }\end{array}$ \\
\hline $\begin{array}{l}\text { Recursos } \\
\text { terapêuticos }\end{array}$ & $\begin{array}{l}\text { - Adriana Rodri- } \\
\text { gues; } \\
\text { - Marisa Mancini; } \\
\text { - Marina Palhares }\end{array}$ & $\begin{array}{l}\mathrm{n}=2 \\
\text { - Atendimentos individuais ou em } \\
\text { grupo; }\end{array}$ & $\begin{array}{l}\mathrm{n}=3 \\
\text { - Estudo observacional; } \\
\text { - Ensaios clínicos com } \\
\text { individuo com seu próprio } \\
\text { controle; } \\
\text { - Registro a partir de ima- } \\
\text { gens de vídeo e fotografia. }\end{array}$ & $\begin{array}{l}\mathrm{n}=1 \\
\text { - Apresentação de considerações de } \\
\text { autores sobre o uso de diferentes } \\
\text { tipos de órteses, em relação ao padrão } \\
\text { patológico das crianças com paralisia } \\
\text { cerebral. }\end{array}$ \\
\hline
\end{tabular}

${ }^{1}$ Classificação baseada em Vieria e Hossne (2001)

${ }^{2}$ Os estudos teóricos estão apresentados ao longo do texto do artigo.

Por outro lado, Pfeifer et al. (2008) indicam que o terapeuta ocupacional pode aplicar atividades, que além de desenvolver as áreas motoras, sensoriais, cognitivas e sociais, favoreçam o desenvolvimento das áreas de desempenho ocupacional infantil e acrescentem à rotina das crianças momentos prazerosos para tornar crescente a motivação e a possibilidade de adquirir habilidades.

A inclusão escolar foi outro tema bastante abordado entre os trabalhos estudados. Jurdi e Amiralian (2006) apresentam a escola como o lugar adequado para promover o aprendizado formal, além de um espaço de trocas e de transmissão de valores sociais e culturais, onde a desmistificação da deficiência pode ocorrer de forma concreta, sendo assim, favorável a todos os atores envolvidos. As autoras ponderam que apesar da intensificação das discussões a respeito desse tema, ainda são encontradas, no cotidiano escolar, inúmeras dificuldades e questões que devem ser esclarecidas (JURDI; AMIRALIAN, 2006).g O professor preparado para a inclusão deve ser capacitado para diferentes estratégias pedagógicas, possíveis de serem aplicadas com diferentes alunos, independentemente se a criança tem deficiência ou não (ROCHA et al., 2003).

Nesse sentido, o trabalho da terapia ocupacional, que pode ser desenvolvido com os educadores, os alunos, os pais e a comunidade é o de facilitação do aparecimento das dificuldades, dos sentimentos e das emoções, que permeiam o relacionamento desses atores com a proposta da inclusão, desvelando os sentidos da deficiência para todos 
e debatendo aspectos do imaginário social do grupo. $\mathrm{O}$ terapeuta ocupacional ainda pode intervir sobre as barreiras arquitetônicas e o mobiliário, fazer adaptações de materiais, utilizar tecnologia assistiva e comunicação alternativa. (ROCHA et al., 2003)

Os artigos que descrevem modelos e instrumentos de avaliação em terapia ocupacional relatam a proposição e o estudo da eficácia de testes de avaliação, bem como estudos sobre grupos infantis atendidos pela T.O. Entre esses artigos, Magalhães et al. (2004) relatam os processos de criação da Avaliação da Coordenação de Destreza Motora, o ACOORDEM, instrumento desenvolvido considerando as dificuldades do uso de testes importados e a falta de recursos disponíveis para a avaliação do desenvolvimento motor de crianças brasileiras. Os autores buscaram criar um teste para detecção de problemas de coordenação motora em crianças entre 4 e 8 anos de idade. A versão final do ACOORDEM, apresentada no artigo, propõe atividades para observação e questionários para a avaliação divididos em três áreas: coordenação e destreza manual, coordenação corporal e planejamento motor e desempenho funcional em casa e na escola.

Pfeifer et al. (2008) discutem o uso de atividades lúdicas na avaliação psicomotora de crianças pré-escolares, com proposição de brincadeiras para avaliar aspectos psicomotores dessas crianças baseando-se na Escala Psicomotora de Vitor da Fonseca (FONSECA, 1995 apud PFEIFER et al., 2008). Os autores observaram que a utilização das atividades lúdicas proporcionou mais espontaneidade e motivação nas ações das crianças, o que torna os dados coletados mais fidedignos.

Finalmente, Moura et al. (2004) utilizam dois instrumentos de avaliação para verificar a evolução do tratamento, bem como a eficácia dos próprios instrumentos: o Gross Motor Function Measure (GMFM), e o Pediatric Evaluation of Disability Inventory (PEDI). O primeiro instrumento foi desenvolvido por Russel e colaboradores, em 1993, com o objetivo de quantificar mudanças na função motora grossa de crianças com paralisia cerebral. O PEDI, criado em 1992, por Haley et al., mensura habilidades funcionais, assistência do cuidador e modificações nas áreas de autocuidado, mobilidade e função social em crianças de 6 meses a 7 anos e meio de idade. Os autores concluem ser vantajoso utilizar os dois instrumentos, que são complementares e permitem a comparação de dados, entre o cliente e um grupo normativo e entre o cliente em momentos diferentes da intervenção. (MOURA et al., 2004).

Alguns outros modelos de intervenção e instrumentos de avaliação foram apresentados: Escala Lúdica Préescolar Knox (PFEIZER; EUFRAZIO, 2006); Guia Portage de educação pré-escolar (VITTA, 2001); Self-Efficacy Gauge (GALVÃO et al., 2008); Erhardt Developmental Vision Assessment - Short (REGOLIN et al., 2006), Modelo Lúdico (ZEN; OMAIRI, 2009), entre outros. É possível notar que não há uma tendência única de uso de modelos e instrumentos de avaliação.

Outra problemática relevante é apresentada em artigos sobre o brincar. Pfeifer e Eufrasio (2006) discutem que o papel ocupacional da criança é o de "brincador". O brincar possibilita que a criança se insira no mundo, fale de si, se desenvolva, absorva conhecimento e aprendizagens, expresse desejos e angústias, de uma forma prazerosa, que possibilita a entrada e manutenção na realidade social.

Jurdi (2001) complementa que a brincadeira é um processo de relações interpessoais, portanto, de cultura e que o brincar faz parte de um aprendizado. No entanto, para as crianças com deficiência a atividade lúdica aparece alterada ou inexistente. Há um sentimento na família da criança de que ela é incapaz e essa falta de expectativas e perspectivas implica em dificuldade de propiciar à criança trocas saudáveis e o desenvolvimento do brincar. Quando o brincar autônomo não é possível, o trabalho do terapeuta ocupacional orienta-se no sentido de indicar à criança e sua família oportunidades para a aquisição da atividade lúdica, a partir de brincadeiras prazerosas e convidando-os a brincar (TAKATORI, 2001). A atividade lúdica pode mostrar a existência da criança, não apenas de sua deficiência ou incapacidade, mas de um ser de desejos e linguagens próprios (JURDI, 2001).

Outros recursos terapêuticos foram apresentados pelos autores. Dois artigos desenvolveram estudos sobre o uso de órteses para melhorar o desempenho funcional da criança e facilitar a realização de algumas atividades. Aguiar e Rodrigues (2002) discutem que para as crianças com paralisia cerebral, as órteses proporcionam melhora da qualidade do movimento do membro plégico e da funcionalidade manual, diminuição do tono muscular, aumento da amplitude dos movimentos e do crescimento muscular, diminuição do padrão patológico e redução de ocorrência de contraturas. Mancini et al. (2007) acrescentam que as órteses devem ser usadas e desempenham melhor função quando complementam outras ações terapêuticas.

Antunes e Vicentini (2005) descrevem a experiência da criação do "tapete sensorial", construído de diferentes texturas, que desenvolve a sensibilidade tátil plantar de crianças com autismo, baseado nos princípios da Terapia de Integração Sensorial, proposta por Ayres (AYRES, 1989 apud ANTUNES; VICENTINI, 2005). Os autores concluíram que com o uso do tapete, ocorreu uma organização de processos neurológicos, com novas aprendizagens, com- 
portamentos estáveis, o que contribuiu para a organização geral do comportamento da criança e de sua interação com o meio ambiente.

Por fim, Palhares et al. (2006) relatam ainda o uso do computador como recurso terapêutico, visando a inclusão, a comunicação e/ou a aprendizagem de crianças com deficiência, a partir de jogos educativos e de caráter lúdico. Recursos de acessibilidade podem ser utilizados para maior independência do usuário, como adaptações no mouse, nos mobiliários e no recurso de acessibilidade de editores de texto. Os autores apontam que o computador pode ser um importante instrumento para ampliar conquistas do ser humano no que tange aos aspectos físico, cognitivo e sensorial, além de um importante instrumento comunicação.

\section{CONCLUSÃO}

A análise da produção bibliográfica de artigos de terapia ocupacional junto à população infantil possibilitou a visualização de um panorama geral dessa prática a partir do olhar e das estratégias de estudo escolhidas pelas autoras desse artigo.

Embora ainda bastante vinculada às instituições de ensino e concentrada na região sudeste, é possível verificar um sensível crescimento nessa produção nos últimos anos. Os trabalhos descritos são desenvolvidos nas áreas de intervenção de saúde, de educação e de assistência social e ainda são escassos aqueles relacionados à atenção básica à saúde, onde a população infantil tem prioridade de acompanhamento No entanto, a atuação e pesquisa de terapeutas ocupacionais junto às crianças, tanto nas Unidades Básicas de Saúde, na Estratégia de Saúde da Família como nos Núcleos de Apoio à Saúde da Família (NASF) pode se configurar como um importante campo. O terapeuta ocupacional pode contribuir para o desenvolvimento de propostas de promoção e manutenção da saúde, prevenção de doenças e cuidados para tratamento e reabilitação, participando de iniciativas assistenciais e de pesquisa sobre processos de intervenção que, inclusive, empreguem recursos e atividades com utilização de baixa densidade tecnológica, próximas do território de moradia e das famílias das crianças.

As problemáticas e/ou temas de estudo elencados na produção bibliográfica estudada demonstram que há um grande campo de trabalho com a população infantil. A atenção à criança em situação de risco social, à criança em processo de adoecimento e hospitalização, ou ainda a inclusão escolar, os modelos e avaliações para intervenção, assim como o estudo sobre o brincar se apresentaram como tendências tanto na pesquisa como na prática dos profissionais, que se dispuseram a divulgar suas experiências.

Por fim, é essencial que os terapeutas ocupacionais invistam na responsabilidade da produção científica e que divulguem os trabalhos por eles desenvolvidos, para o crescimento e fortalecimento da profissão.

GOMES, M. L.; OLIVER, F. C. The practice of Occupational therapy with the child population: literature review of the period from 1999 to 2009. Rev. Ter. Ocup. Univ. São Paulo, v. 21, n. 2, p. 121-129, maio/ago. 2010.

\begin{abstract}
This article is on the work developed by occupational therapists with the children in Brazil. A literature review of articles produced between 1999 and 2009 was conducted in the following databases: Latin-American and Caribbean Literature of Health Sciences, Scientific Electronic Library Online, and the periodic Cadernos de Terapia Ocupacional da UFSCar. Data were analyzed based on authorship, year, periodical, kind of work, methodology/model of attention, problematic and institution/place of work. 84 articles were identified, most of which were productions linked to universities, especially from the Southeast. The works with children are developed within the fields of health, education and social assistance. Experience reports and research articles were among the most common type of articles found. The methodologies employed in research studies are usually observational and descriptive studies and clinical trials. The interventions listed in the experience reports were individual and group care and the use of leisure activities, among others. Among the 12 issues listed, the most studied were the attention to children in social risk situation and in process of illness and hospitalization, school inclusion, playing, models and instruments of evaluation and activities and therapeutic resources. The study allowed an overview of occupational therapists' work with children from the viewpoint and strategies chosen by the authors in their studies.
\end{abstract}

KEY WORDS: Occupational therapy/trends; Occupational therapy/methods; Review literature as topic; Practice guideline; Child; Child development; Child advocacy. 


\section{REFERÊNCIAS}

AIRÉS, P. História social da criança e da família. Rio de Janeiro: Ed. Guanabara, 1981.

AGUIAR, I. F.; RODRIGUES, A. M. V. N. O uso de órteses no tratamento de crianças com paralisia cerebral na forma espástica:uma revisão bibliográfica. Temas sobre Desenvolvimento, v. 11, n. 63 , 2002.

ANTUNES, E. S. C. F.; VICENTINI, C. R. Desenvolvendo a sensibilidade sensorial tátil plantar em portadores de autismo infantil através do "tapete sensorial" - estudo de três casos. Cad. Ter. Ocup. UFSCar, v. 13, n. 1, 2005.

AOKI, M.; OLIVER, F. C.; NICOLAU, S. M. Pelo direito de brincar. Rev. Ter. Ocup. Univ. São Paulo, v. 17, n. 2, p. 57-63, 2006.

BARTAlOTTI, C. C.; CARLO, M. M. R.P. (Org.). Terapia ocupacional no Brasil: fundamentos e pespectivas. São Paulo, Plexus Editora. 2001.

BARROS, D. D.; GHIRARDI, M. I. G.; LOPES, R. E. Terapia ocupacional social. Rev. Ter. Ocup. Univ. São Paulo, v. 13, n. 3, p. 95-103, 2002.

BARROS, D. D. Terapia ocupacional no território: as crianças e os adolescentes da unidade do Brás - movimento de luta de moradia urbana. Cad. Ter. Ocup. UFSCar, v. 9, n. 1, 2001.

CAVALCANTI, A.; GALVÃO, C. R.C. Terapia ocupacional: fundamentação \& prática. Rio de Janeiro: Guanabara Koogan, 2007.

EL-KATIB, U. Um estudo acerca da passagem de crianças e adolescentes pelo judiciário no município de São Carlos. Cad. Ter. Ocup. UFSCar, v. 13, n. 1, 2005.

GALHEIGO, S. M.; RUSSO, J. A. A caracterização da produção bibliográfica nas práticas hospitalares em terapia ocupacional no Brasil: uma revisão na literatura de 1990 a 2007. Rev. Ter. Ocup. Univ. São Paulo, v. 19, n. 2, p. 91-99, 2008.

GALHEIGO, S. M.; ANGELI, A. A. C. Terapia ocupacional e o cuidado integral à saúde de crianças e adolescentes: a construção do Projeto ACCALANTO. Rev. Ter. Ocup. Univ. São Paulo, v. 19, n. 3, 2008.

GAlVÃO, B. A. P.; LAGE, N. V.; RODRIGUES, A. A. C. Transtorno do desenvolvimento da coordenação e senso de autoeficácia: implicações para a prática da terapia ocupacional. Rev. Ter. Ocup. Univ. São Paulo, v. 19, n. 1, p. 12-19, 2008

JURDI, A. P. S. Atividade lúdica: uma atividade criativa. Temas sobre Desenvolvimento, v. 10, n. 56, 2001.

JURDI, A. P. S.; AMIRALIAN, M. L. T. M. A inclusão escolar de alunos com deficiência mental: uma proposta de intervenção do terapeuta ocupacional no cotidiano escolar. Estudos Psicol. (Campinas), v. 23, n. 2, 2006.

KUDO, A. M.; MARCONDES, E.; LINS, M. L. F.; MORIYAMA, L. T.; GUIMARAES, M. L. L. G.; JULIANI, R. C. T. P.; PIERRI, S. A. (Org). Fisioterapia, fonoaudiologia e terapia ocupacional em pediatria. São Paulo: Sarvier, 1990.
MAGALHÃES, L. C.; NASCIMENTO, V. C. S.; REZENDE, M. B. Avaliação da coordenação e destreza motora - ACOORDEM: etapas de criação e perspectivas de validação. Rev. Ter. Ocup. Univ. São Paulo, v. 15, n. 1, 2004.

MALFITANO, A. P. S.; BRAGA, I. F.; SILVIA, K. G.; MOTA, N. G. A promoção de direitos de crianças e adolescentes em situação de vulnerabilidade social: oficina de brincadeiras como recurso. Cad. Ter. Ocup. UFSCar, v. 14, p. 103-110, 2006.

MANCINI, M.C.; RODRIGUES, A. M.; VALLADÃO N.; VAZ, D. V.; SILVA, L.C. Uso de órtese para abdução do polegar no desempenho funcional de criança portadora de paralisia cerebral: estudo de caso único. Rev. Bras. Saude Mater. Infant., v. 7, n. 4, 2007.

MOURA, E. W.; MAKITA, L. M.; OLIVEIRA, M. C. Utilização de pedi e gmfm no planejamento de tratamento de criança com paralisia cerebral do tipo atetóide e paralisia braquial obstétrica. Temas sobre Desenvolvimento, v. 13, n. 73, 2004.

PALHARES, M. S.; MARINO, A. E. E.; LIMA, F. C. Iniciação ao uso do computador: um relato de experiência com crianças com paralisia cerebral. Cad. Ter. Ocup. UFSCar, v. 14, n. 1, 2006.

PFEIFER, L. I. TATSUGUCHI, J. M.; SILVA, L. C. R.; EUFRAZIO, M. C. Atividades lúdicas na avaliação psicomotora de pré-escolares. Temas sobre Desenvolvimento, v. 16, n. 191, 2008 .

PFEIFER, L. I.; EUFRAZIO, M. C. Influência do brincar para o desenvolvimento de crianças institucionalizadas de 3 a 6 anos. Temas sobre Desenvolvimento, v. 15, n. 85-86, 2006.

PFEIFER, L. I.; GRIGOLATTO, T.; CHAVES, G. F.; SILVA, M. B. Intervenção terapeutica ocupacional em cti pediatrico: um estudo de caso. Cad. Ter. Ocup. UFSCar, v. 16, n. 1, 2008.

REGOLIN, K.;FRANÇA, L.; JIMENEZ, L.; SAKOMOTO, R. H.; KAMIDA, N. T. S.; MATHEUS, K. R. M.; KAWAKAMI, R.; MARTINI, G.;OLIVEIRA, M. C.; ZERBINATO, L. Avaliação da função visual em crianças com paralisia cerebral tipo tetraparesia e diparedia espástica: apresentação de um instrumento em terapia ocupacional. Temas Desenvolvimento, v. 15, n. 85-86, 2006.

ROCHA, E. F.; LUIZ, A.; ZULIAN, M. A. R. Reflexões sobre as possíveis contribuições da terapia ocupacional nos processos de inclusão escolar. Rev. Ter. Ocup. Univ. São Paulo, v. 14 n. 2,2003 .

TAKATORI, M.; BOMTEMPO, E.; BENETTON, M. J. O brincar e a criança com deficiência física: a construção inicial de uma história em terapia ocupacional. Cad. Ter. Ocup. UFSCar, v. 9, n. 2, 2001.

VIEIRA, S.; HOSSNE, W. S. Metodologia científica para a área da saúde. Rio de Janeiro: Campos, 2001.

VITTA, F. C. F. Avaliação terapêutica ocupacional de crianças com encefalopatias crônicas infantis não progressivas. Cad. Ter. Ocup. UFSCar, v. 9, n. 2, 2001.

ZEN, C. C.; OMAIRI, C. O modelo lúdico: Uma nova visão sobre o brincar para a terapia ocupacional. Cad. Ter. Ocup. UFSCar, v. 17, n. $1,2009$. 